\title{
CMC-Bauteile für Heißgasanwendungen: Von der Entwicklung des Prototypen bis hin zum Serienbauteil
}

\author{
Martin Frieß, Christian Zuber, Severin Hofmann, Matteo Crippa, Bernhard Heidenreich \\ Deutsches Zentrum für Luft- und Raumfahrt (DLR), Institut für Bauweisen- und Konstruktionsforschung, Pfaffenwald- \\ ring 38 - 40, 70569 Stuttgart
}

\section{$1 \quad$ Einleitung und Zielsetzung}

Faserverstärkte keramische Verbundwerkstoffe (CMC) vereinen die generellen Eigenschaften keramischer Werkstoffe (Abrasions- und Temperaturbeständigkeit, -standfestigkeit sowie hohe Härte) mit den positiven Eigenschaften faserverstärkter Verbundwerkstoffe (hohe Schadenstoleranz im Überlastfall). Aufgrund ihrer dadurch bedingten extremen Thermoschockbeständigkeit und Temperaturstandfestigkeit bis über $2000{ }^{\circ} \mathrm{C}$ sind sie ideale Kandidaten für Heißgasanwendungen in der Luft- und Raumfahrt, z.B. für Raketenantriebe [1,2].

Das vom DLR entwickelte Flüssigsilicier-Verfahren (LSI) bietet nahezu ideale Voraussetzungen, um kostengünstig faserkeramische Bauteile auf der Basis von $\mathrm{C} / \mathrm{C}$-SiC herstellen zu können, die aufgrund ihrer Bauteilgeometrie mit anderen CMC-Herstellverfahren (z.B. CVI) nicht realisierbar sind [3-8].

In diesem Beitrag wird über die Entwicklung und Herstellung von Bauteilen zur Steuerung von Raketenantrieben berichtet: Prototypen-Entwicklung bis hin zu zertifizierten Serienbauteilen. Dazu wurden in Zusammenarbeit mit dem Industriekunden fast 120 Bauteile nach einer applikationssowie entwicklungsbedingten Vorgabe gefertigt und prozessbegleitend, zerstörend an bauteiltypischen Proben sowie zerstörungsfrei an den eingesetzten Bauteilen mittels Computertomographie sowie Porositäts- und Dichtemessungen qualifiziert. Aufgrund sich ändernder Rohstoffqualitäten und zur Verbesserung des Produkts $\mathrm{C} / \mathrm{C}$-SiC mussten die CFK-Herstellungsparameter angepasst werden. Es soll auch ein statistisch belastbarer Zusammenhang verschiedener Eigenschaften der Ausgangsrohstoffe und Prozessparameter auf die Werkstoffeigenschaften aufgezeigt werden.

\section{Experimentelles}

Das Herstellungsschema für C/C-SiC Verbundwerkstoffe via LSI-Prozess ist in Abb.1 skizziert. Aus Festigkeits- und Rentabilitätsgründen wurde das Autoklav-Verfahren ausgewählt. Als Ausgangsmaterial wurde nach DLR-Vorgaben hergestelltes, mit Phenolharz vorimprägniertes handelsübliches C-Gewebe (HTA, Fa. Tenax) auf der Basis von PAN (HTA-Prepregs) eingesetzt, das in Leinwandbindung $\left(240 \mathrm{~g} / \mathrm{m}^{2}\right)$ erhältlich war. Zur Verbundwerkstoffherstellung wurden die auf 200 x $250 \mathrm{~mm}$ zugeschnittenen 169 HTA-Prepreg-Lagen nach einem vorgegebenen Lagenaufbau aufeinander drapiert, wobei aus Festigkeitsgründen Kett- auf Kettfaden in der Hauptbelastungsrichtung lagen. Aus Steifigkeits- und Symmetriegründen wurden im Kern mehrere $+/-45^{\circ}$-Lagen eingebaut.

Für die Prozessoptimierung der CFK-Herstellung via Autoklav-Verfahren wurde zunächst an einer einzelnen Platte, anschließend an zwei Platten und dann an vier Platten ein spezieller Prozess- 
zyklus entwickelt. Dazu wurden Temperatur und Druck sowie Setzweg und Faservolumengehalt bestimmt und untereinander verglichen. Anschließend wurden die Platten randseitig beschnitten und bei $240{ }^{\circ} \mathrm{C}$ an Luft über einen Zeitraum von $23 \mathrm{~h}$ getempert.

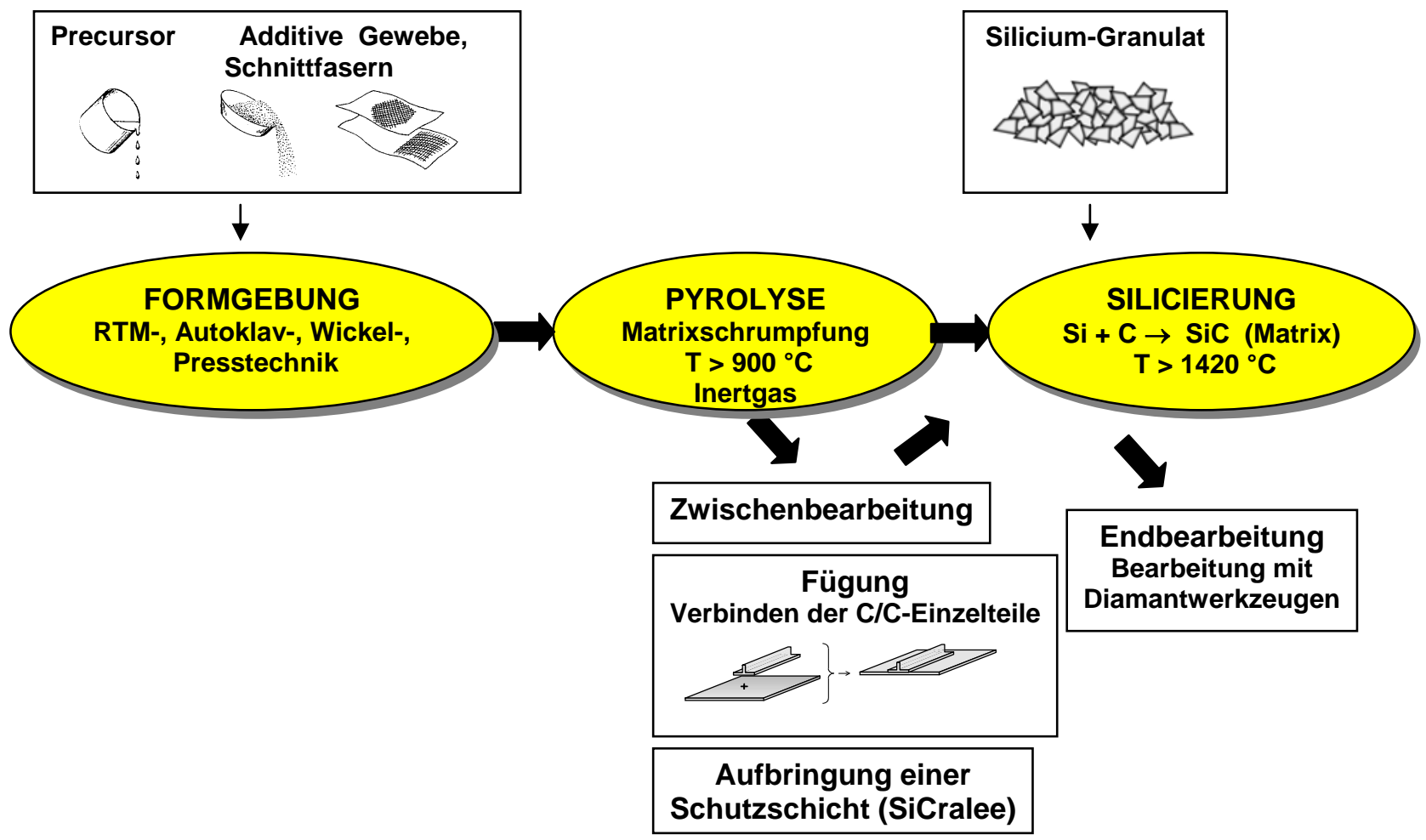

Abb.1: Herstellungsschema für C/C-SiC Verbundwerkstoffe nach dem LSI-Verfahren.

Die so hergestellten, $41 \mathrm{~mm}$ dicken CFK-Platten wurden zunächst in $\mathrm{N}_{2}$-Atmosphäre bis $900{ }^{\circ} \mathrm{C}$ anschließend bei ca. 1 mbar bis $1650{ }^{\circ} \mathrm{C}$ zum C/C-Werkstoff pyrolysiert. Danach erfolgte der $\mathrm{Zu}-$ schnitt der Bauteilrohlinge sowie der Rohlinge für die Kurzbiegeproben via Wasserstrahlschneiden. Nach dem Trocknen der Bauteile bei $110{ }^{\circ} \mathrm{C}$ wurden die für den nächsten Prozessschritt (Silicierung) erforderlichen Parameter offene Porosität und Dichte via Archimedes-Verfahren (Immersion in Wasser nach DIN EN 993-1) ermittelt.

Im nächsten Schritt wurden die C/C-Rohlinge (Bauteile und Kurzbiegeproben) im Vakuum bei einer Maximaltemperatur von $1650{ }^{\circ} \mathrm{C}$ mit flüssigem Silicium infiltriert und zu C/C-SiC-Bauteilen konvertiert.

Anschließend erfolgte die mechanische Endbearbeitung der Bauteilgeometrie innerhalb enger, Toleranzen. Zur Ermittlung der mechanischen Eigenschaften, wie Biege-, Zug- und Druckscherfestigkeit wurden die Proben nach einem definierten Probenplan mit Diamantwerkzeugen aus den C/CSiC-Rohlingen herausgearbeitet (Tab.1). So wurden beispielsweise für die Kurzbiegeproben (35 x $10 \times 5 \mathrm{~mm}^{3}$ ) jeweils 3 Proben oberhalb und 3 Proben unterhalb der Symmetrielagen in Kettrichtung aus der Platte herausgearbeitet.

Nach dem Reinigen der Bauteile in deionisiertem Wasser mittels Ultraschallbad wurde die offene Porosität und Dichte via Archimedes nach DIN ermittelt.

Anschließend wurden die Bauteile mittels Computertomografie (3 Stück in einer Messung) auf mögliche Bauteilfehler untersucht. 
Tab.1: Ermittlung der mechanischen Eigenschaften nach EN-V-Norm.

\begin{tabular}{lcllll}
\hline Versuch & EN-V-Norm & $\begin{array}{l}\text { Probengeometrie } \\
{[\mathrm{mm}]}\end{array}$ & $\begin{array}{l}\text { Auflagerabstand } \\
{[\mathrm{mm}]}\end{array}$ & $\begin{array}{l}\text { Orientierung } \\
\text { zum Schaft }\end{array}$ & Eigenschaft \\
\hline Kurzbiege- & $658-5$ & $\begin{array}{l}35 \times 10 \times 5 \\
3 \text { oben }+3 \text { unten }\end{array}$ & $25(1 / \mathrm{d}=5)$ & $\|+\perp$ & Biegefestigkeit \\
Langbiege & $658-3$ & $\begin{array}{l}110 \times 10 \times 5 \\
\text { 3 oben }+3 \text { unten }\end{array}$ & $100(1 / \mathrm{d}=20)$ & $\|+\perp$ & Biegefestigkeit \\
Zug- & $658-1$ & $\begin{array}{l}130 \times 10 \times 3 \\
2 \text { oben }+2 \text { unten }\end{array}$ & - & $\|$ & Zugfestigkeit \\
Druck- & $60 \times 20 \times 15$ & 10 & $\|$ & $\begin{array}{l}\text { Druckscher- } \\
\text { Scher- }\end{array}$ \\
\hline
\end{tabular}

\section{Ergebnisse und Diskussion}

\subsection{Verbundwerkstoffherstellung}

Bei der Entwicklung der CFK-Herstellung wurde der Einfluss des Harzgehalts des Prepregs als kritischer Faktor identifiziert, der umfangreiche Prozessparameteranpassungen erfordert. Ein zu hoher Harzgehalt führt zu geringeren Faservolumengehalten und erschwert die Einhaltung des geforderten Faservolumengehalts (54-60 Vol-\%), ein zu geringer Harzgehalt bedingt ein poröses Laminat, das eine erhöhte Si-Aufnahme während der Silicierung sowie verringerter Festigkeitswerte zur Folge hat. Daher sollte ein bevorzugter Harzgehalt zwischen 38 und $42 \%$ liegen.

Des Weiteren zeigte sich, dass der entwickelte Temperprozess unbedingt eingehalten werden muss, da bei Nichteinhaltung die Festigkeitswerte signifikant erniedrigt und hohen Schwankungen unterworfen sind (Abb.4).

Der Pyrolyseprozess gestaltete sich aufgrund seiner konservativen Auslegung als unkritisch. Die gemessenen offenen Porositäten und Dichten wiesen, abgesehen von der erhöhten Dichte bei den vorausgegangenen inkorrekten Bedingungen beim Temperprozess keine Besonderheiten auf.

Delaminationsneigungen während des Wasserstrahlschneidens der C/C-Rohlinge aus den C/CPlatten traten sehr selten auf, sofern die vorgeschriebenen Parameter eingehalten wurden. Während des Silicierprozesses traten keine Schwierigkeiten auf, solange die Si-Zugabe eingehalten wurde. Es konnten zu keiner Zeit Delaminationsneigungen festgestellt werden, die zu einem Ausschlusskriterium führten.

Die kundenspezifisch entwickelte, mechanische Bearbeitung von C/C-SiC-Rohlingen sowie Kurzbiegeproben mittels Diamantwerkzeugen erwies sich aufgrund langjähriger Erfahrung von DLR-Mitarbeitern als sehr robust.

Es wurden sehr wenige C/C-SiC-Bauteile außerhalb der kundenspezifisch geforderten Toleranzen gemessen. Die festgelegten Toleranzen von Seiten des Kunden bzgl. Porosität und Dichte wurden von sehr wenigen Ausnahmen (Temperproblem) abgesehen - eingehalten (Abb.4).

\subsection{Verbundwerkstoffeigenschaften}

In Abb.2 ist die typische Mikrostruktur des entwickelten C/C-SiC-Verbundwerkstoffs, die auch dem DLR-Standardwerkstoff XB entspricht, dargestellt: dichte C/C-Faserbündel (dunkelgrau) sind von SiC-Matrix (hellgrau) umgeben, der Anteil an freiem Si liegt unter 5 Masse-\%. 

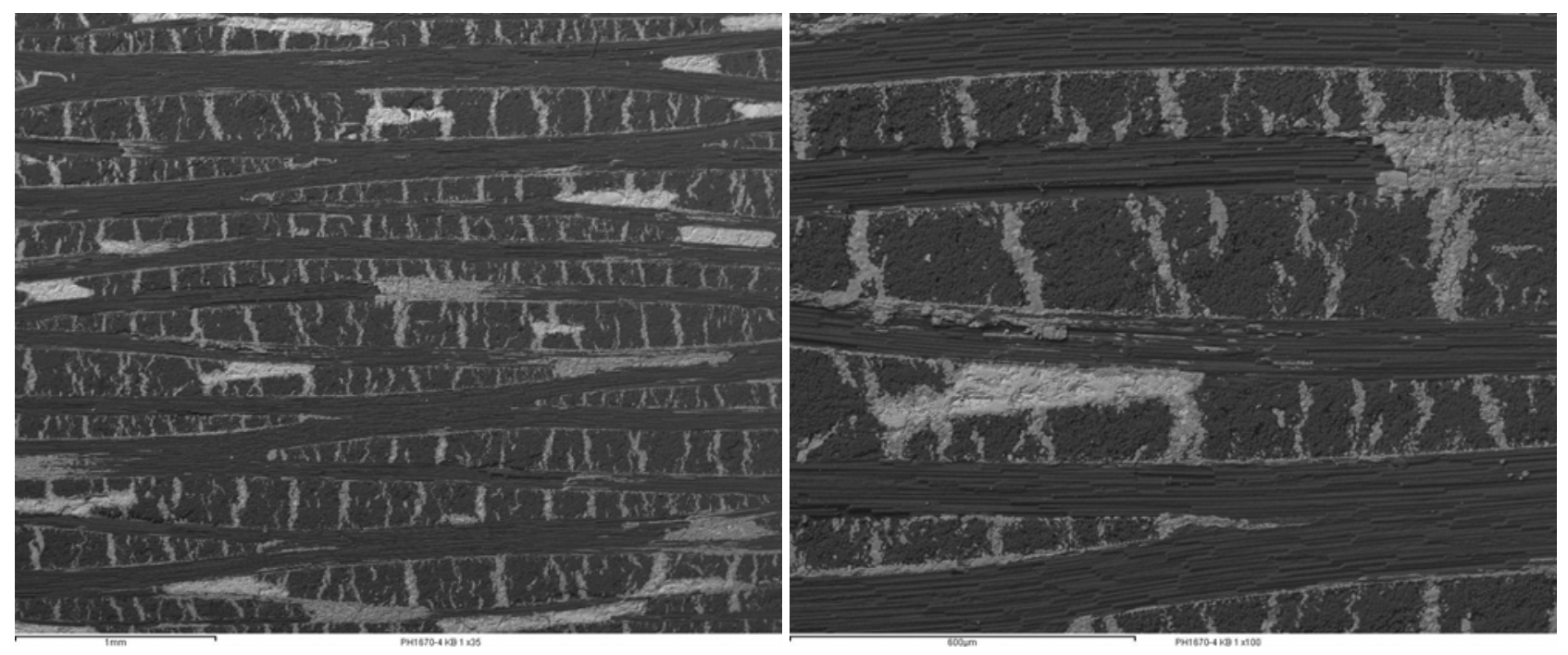

Abb.2: Mikrostruktur (REM-Aufnahme) des C/C-SiC-Verbundwerkstoffes (li.: 35-fache, re.: 100-fache Vergrößerung, dunkelgrau: $\mathrm{C} / \mathrm{C}$, hellgrau: $\mathrm{SiC}$ ).

In einem Qualifizierungsprogramm wurden die mechanischen Eigenschaften, wie Biege-, Zugund Schubfestigkeit, nach den in Tab.1 beschriebenen Methoden ermittelt. Die Ergebnisse sind in Tab.2 dargestellt.

Tab.2: Mechanische Eigenschaften der hier beschriebenen C/C-SiC-Verbundwerkstoffe: Kurzbiege- (KB), Langbiege(LB), Zug- und Schubfestigkeit in paralleler $(\|)$ sowie senkrechter $(\stackrel{\perp}{)}$ Orientierung zum Schaft.

\begin{tabular}{lrrrrrr}
\hline Eigenschaft & $\mathrm{KB}(\|)$ & $\mathrm{KB}(\perp)$ & $\mathrm{LB}(\|)$ & $\mathrm{LB}(\perp)$ & $\mathrm{Zug}(\|)$ & $\operatorname{Schub}(\|)$ \\
\hline Mittelwert [MPa] & 194,8 & 157,5 & 207,8 & 175,1 & 121,2 & 26,6 \\
Standardabweichung [MPa] & 16,7 & 7,5 & 6.5 & 10,7 & 8,1 & 4,8 \\
Plattenanzahl [-] & 6 & 6 & 4 & 6 & 4 & 5 \\
\hline
\end{tabular}

Da das größte Biegemoment der Strahlruder im Einsatzfall in Schaftrichtung ( $\|$ ) vorherrscht und in Kettrichtung höhere Festigkeiten zu erwarten sind, wurden die Kettfäden dementsprechend orientiert. Dies wurde durch die Messwerte bestätigt. Die gemessenen Biegefestigkeiten im Langbiegeversuch sind aufgrund des höheren Schubbelastungsanteils etwas höher als im Kurzbiegeversuch. Gegenüber anderen CMC-Herstellverfahren (z.B.: CVI und LPI) besitzen C/C-SiC-Werkstoffe nach dem LSI-Verfahren sehr hohe Schubfestigkeiten bei gleichzeitig sehr hoher Schadenstoleranz (Abb.5 links).

Aufgrund dieser Eigenschaften wurden in Abstimmung mit dem Kunden für die Herstellung von knapp 120 Strahlrudern unter anderem folgende Qualitäts- bzw. Ausschusskriterien festgelegt: Faservolumengehalt im CFK-Zustand $54-60$ Vol-\%, Kurzbiegefestigkeit $160-200 \mathrm{MPa}$, offene Porosität $0,5-2,5 \%$, Dichte $1,8-2,0 \mathrm{~g} / \mathrm{cm}^{3}$ sowie enge Bauteiltoleranzen und keine sichtbaren Schäden bei der CT-Untersuchung.

Die unter 3.1 Verbundwerkstoffherstellung beschriebene Entwicklung führte zu den in Tab.3 beschriebenen Bauteileigenschaften. Der typische Verlauf der Eigenschaften während der Herstellungsphase ist in Abb.3 gezeigt. In umfangreichen CT-Untersuchungen konnten keine signifikanten Bauteilfehler festgestellt werden (ohne Befund). Dies erwies sich in Kombination mit anderen Methoden als sehr wichtiges NDT-Auschusskriterium. 
Tab.3: Eigenschaften der C/C-SiC-Verbundwerkstoffe in der Herstellungsphase (ohne Bauteile aus fehlerhaftem Temperprozess).

\begin{tabular}{llll}
\hline Eigenschaft & Offene Porosität [\%] & Dichte $\left[\mathrm{g} / \mathrm{cm}^{3}\right]$ & KB-Festigkeit [MPa] \\
\hline Mittelwert & 1,052 & 1,940 & 204,1 \\
Standardabweichung & 0,261 & 0,027 & 12,1 \\
Probenanzahl & 150 & 150 & 78 \\
\hline
\end{tabular}

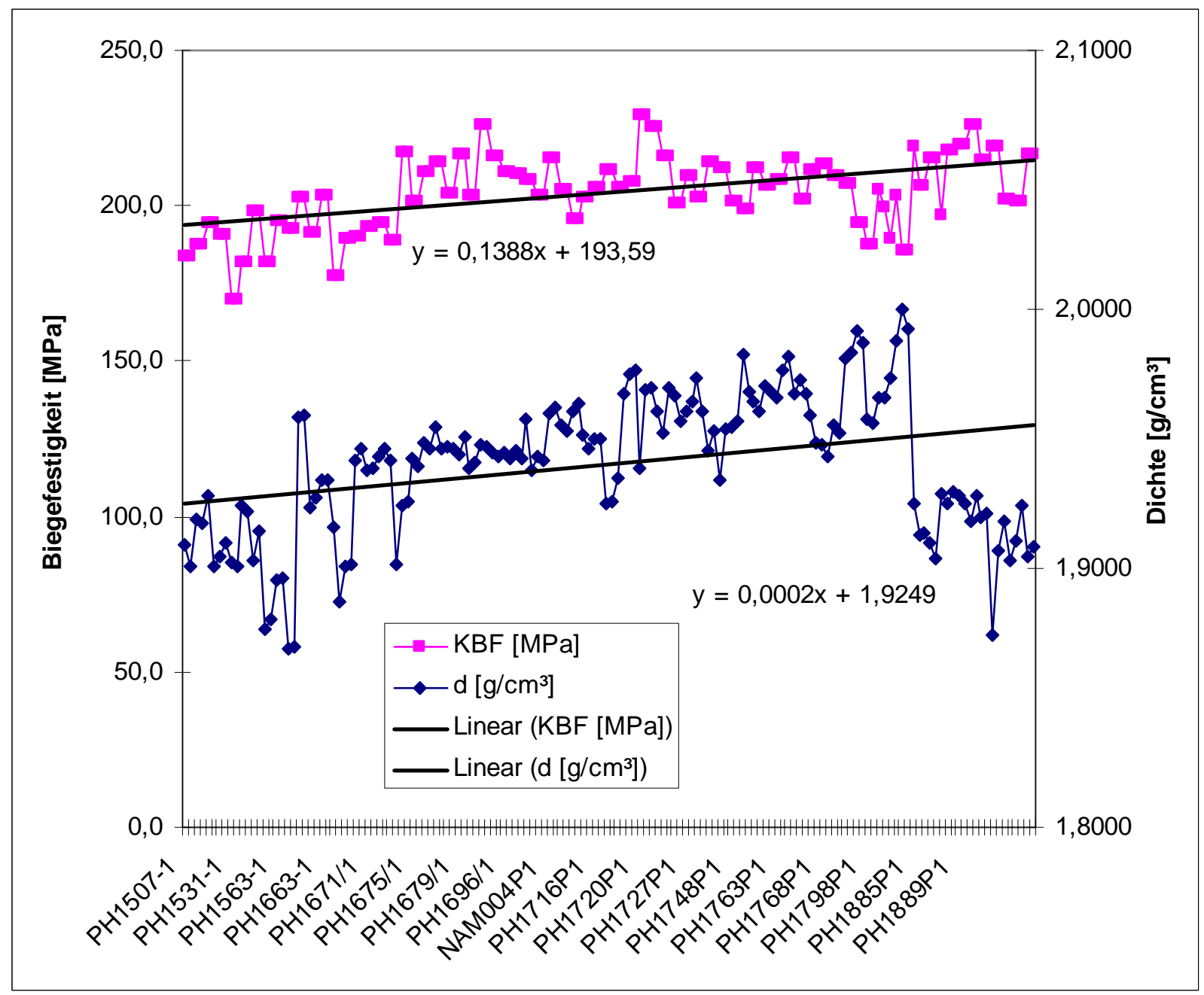

Abb.3: Verlauf der gemessenen Biegefestigkeiten an Kurzbiegeproben sowie der Dichte von Strahlrudern in einem Zeitraum von einem Jahr (ohne Bauteile aus fehlerhaftem Temperprozess).

Während der Herstellungsphase wurden aufgrund eines Fehlers im Tempervorgang bei der CFKHerstellung (defekter Temperofen) sieben Ausschussplatten hergestellt (Abb.4). Dies äußerte sich in signifikant erniedrigten Festigkeitswerten und größeren Risslängen sowie erhöhter Dichte in Verbindung mit einem höheren SiC-Gehalt (Abb.4). Durch die eingeleitete Problemanalyse konnte der Schaden schnell behoben werden. Ein anfänglicher Verdacht auf fehlerhafte Rohstoffe konnte rasch ausgeräumt werden. 


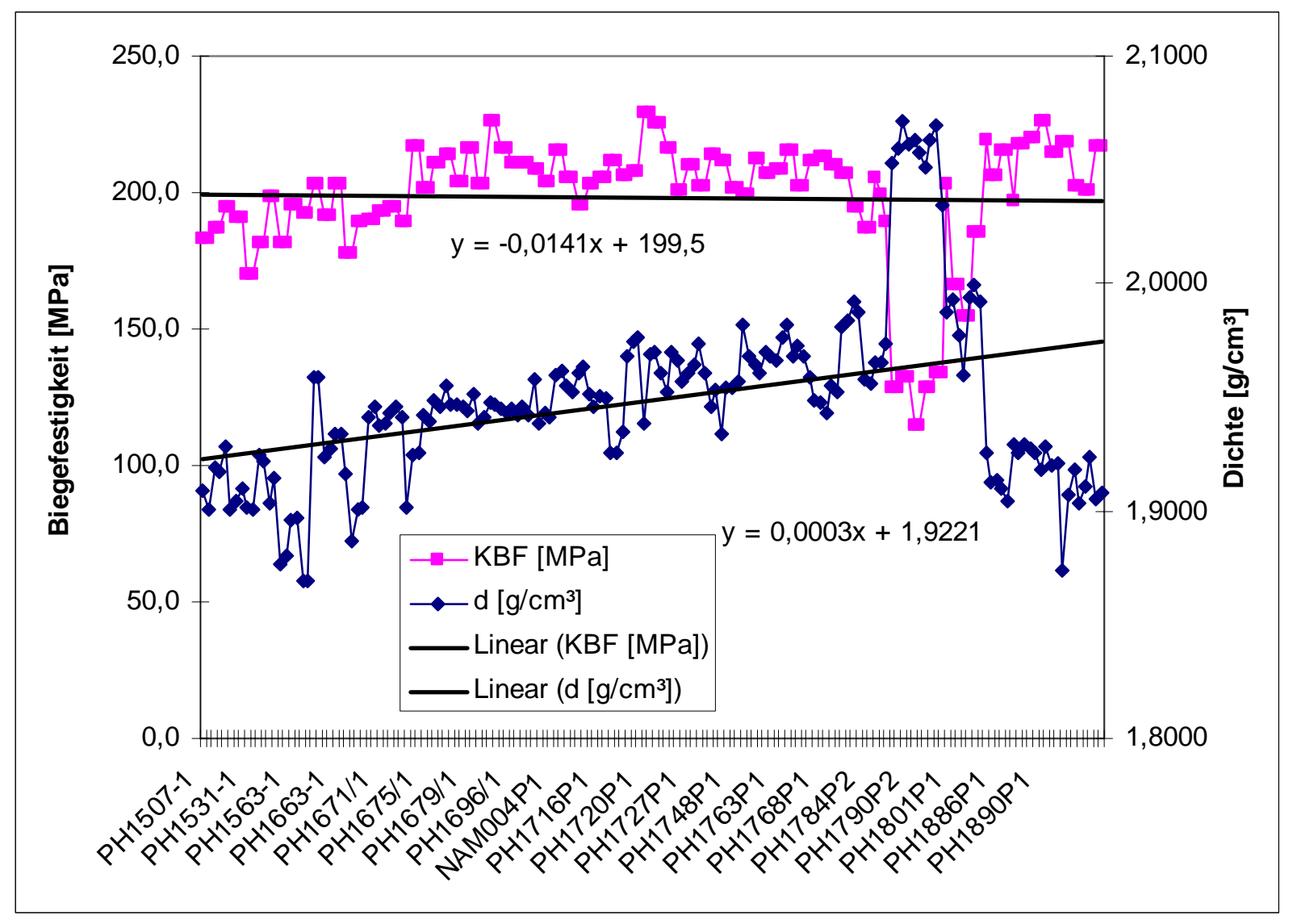

Abb. 4: Verlauf der gemessenen Biegefestigkeiten an Kurzbiegeproben sowie der Dichte von Strahlrudern in einem Zeitraum von einem Jahr. Die niedrigen Festigkeitswerte sowie die erhöhten Dichtewerte sind auf einen fehlerhaften Temperprozess bei der CFK-Herstellung zurückzuführen.
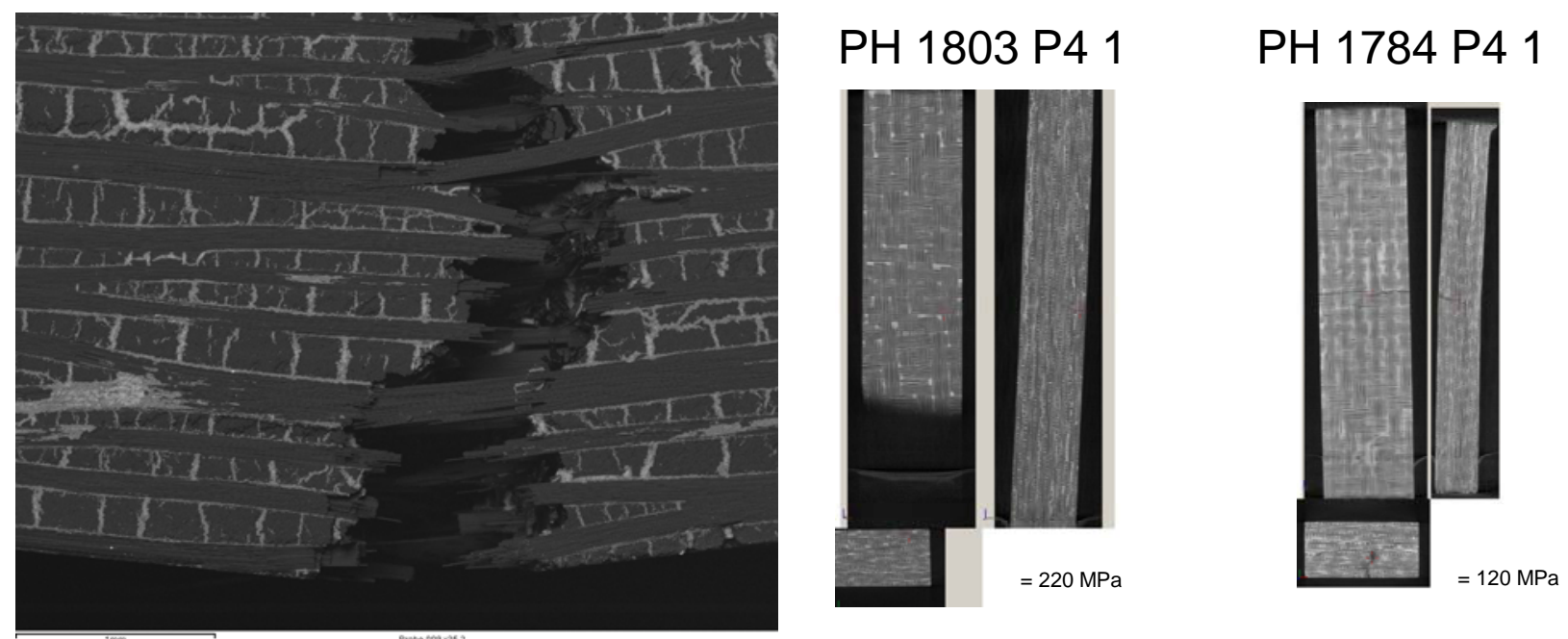

Abb.5: Links: REM-Aufnahme einer im Kurzbiegeversuch gebrochenen C/C-SiC-Probe. Deutlich zu erkennen ist der Pull-out der C-Fasern. Rechts: CT-Aufnahmen an Kurzbiegeproben mit signifikant unterschiedlichen Festigkeiten. Deutlich zu erkennen ist die unterschiedliche Risslänge sowie die Graustufung und damit der SiC-Gehalt (je heller desto höher der SiC-Gehalt). 


\subsection{Anwendung}

In Abb.6 sind einsatzrelevante Bedingungen von C/C-SiC-Strahlrudern dargestellt. Um die Thermoschockbeständigkeit von Strahlrudern oder allgemein C/C-SiC-Bauteile während der Entwicklungsphase zu demonstrieren, wurden exemplarische Strahlruder in einer DLR-eigenen Plasmabeschichtungsanlage erfolgreich getestet (Abb.6 li.).

Ebenfalls in Abb.6 (re.) ist das Ergebnis eines typischen Raketenmotortests mit Einsatztemperaturen $>2000{ }^{\circ} \mathrm{C}$ unter Heißgasbedingungen unter oxidierenden und chlorwasserstoffhaltigen Bedingungen sowie geringem, schub- bzw. verbrennungstechnisch förderndem Aluminiumpartikelzusatz bei einem wenige Sekunden dauerndem Einsatz gezeigt. Strahlruder ermöglichen die Ablenkung des Abgasstrahls bereits in der Start- oder Aufstiegsphase, in der aerodynamische Steuerungsmöglichkeiten wenig wirksam sind, und halten somit die Rakete extrem wendig und manövrierfahig. Der Einsatz von Strahlrudern bei High-Performance-Raketen mittels Schubvektorsteuerung erweist sich als Schlüsseltechnologie.

Die Bedingungen sind extrem. Dennoch erwiesen sich die beim DLR entwickelten C/C-SiCWerkstoffe - insbesondere bei dickwandigen Bauteilen - der weltweiten Konkurrenz überlegen. Die Technologie zur Herstellung der Strahlruder konnte erfolgreich zum Kunden transferiert werden, der inzwischen die Serienproduktion der Strahlruder sowie der hier nicht beschriebenen, am DLR entwickelten Dichtungsringe erfolgreich aufgenommen hat und im Rahmen von NATO-weiten Projekten vermarktet.
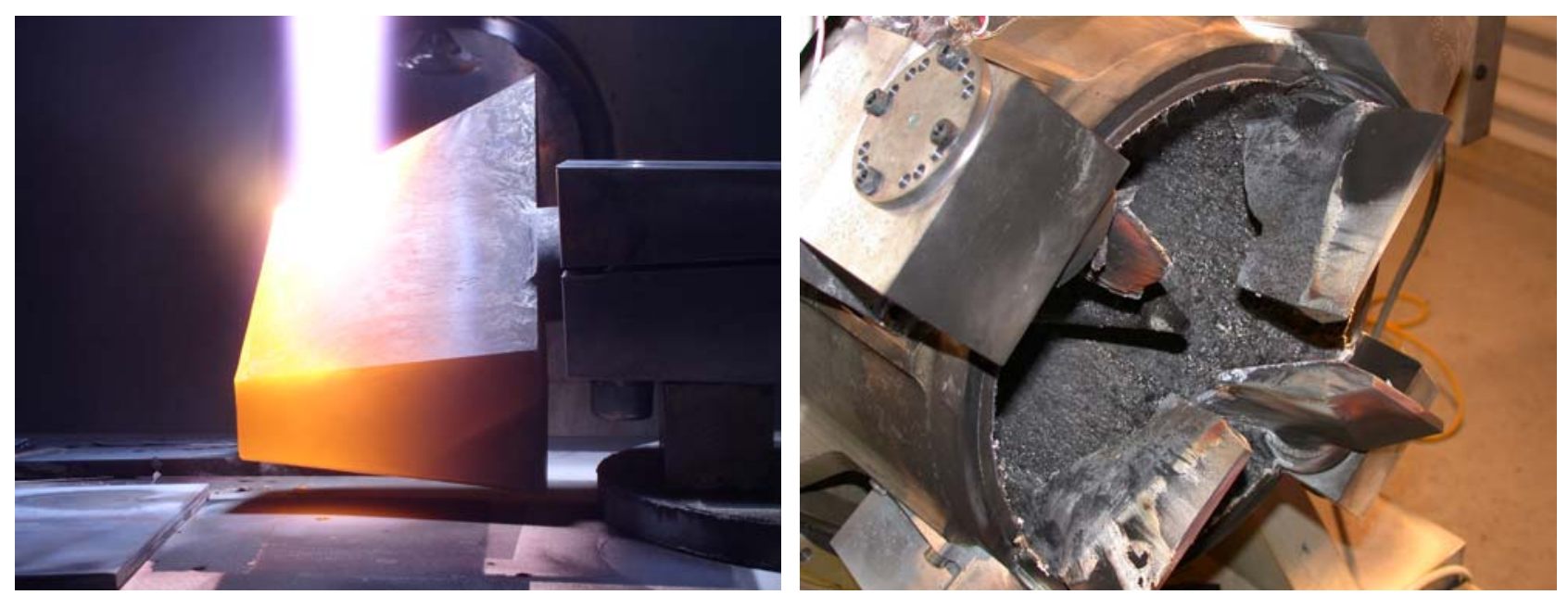

Abb.6: Strahlruder aus C/C-SiC in einer Plasmabeschichtungsanlage des DLR (Thermoschocktest) sowie nach einem typischen Raketenmotortest (Temperaturen $>2000{ }^{\circ} \mathrm{C}$, Heißgas verbrannte Aluminiumpartikel) mit einer Testdauer von wenigen Sekunden.

\section{Zusammenfassung und Ausblick}

Der am DLR in Stuttgart entwickelte LSI-Prozess bietet - unabhängig von der Bauteilgeometrie eine relativ einfache Möglichkeit zur Herstellung von langfaserverstärkten keramischen Verbundwerkstoffen auf der Basis von $\mathrm{C} / \mathrm{C}$-SiC. Aufgrund kundenspezifischer Anforderungen für den Einsatz in den mit Heißgas sowie mit schubfördernden Aluminiumpartikeln beaufschlagten Bauteilen für Raketenmotoren wurde ein für die Serie tauglicher Prozess auf der Basis des AutoklavVerfahrens entwickelt und erfolgreich zum Kunden transferiert. 
In diesem Beitrag konnte zudem gezeigt werden, dass der am DLR entwickelte Prozess zur Kleinserienproduktion von ca. 120 Strahlrudern mit hohen Qualitätsanforderungen erfolgreich eingesetzt werden konnte. Der Prozess erwies sich als sehr robust und aufgetretene Fehler konnten rasch behoben werden. Das ursprünglich in der Entwicklungsphase, festgelegte Biegefestigkeitsniveau von 160 - $200 \mathrm{MPa}$ wurde mit 204,1 MPa im Kurzbiegeversuch sogar leicht überschritten. Die Schwankungen in Festigkeit, aber auch offene Porosität und Dichte hielten sich in engen Grenzen. In CTUntersuchungen konnten keine kritischen Fehler erkannt werden.

Zukünftige High-Performance-Raketen sind ohne Komponenten auf der Basis von CMC (z.B.: $\mathrm{C} / \mathrm{C}-\mathrm{SiC}$ zur Schubvektorsteuerung) schwer zu realisieren. Des Weiteren ist die Integration alternativer Techniken (Wickel- und Flechttechnik) im LSI-Prozess unabdingbar.

\section{$5 \quad$ Danksagung}

Für die tatkräftige Unterstützung bei diesem Projekt möchte ich mich bedanken bei: Markus Keck,, Jürgen Krapf, Stefan Frick, Felix Vogel, Matthias Scheiffele, Ingo Fischer, Raouf Jemmali, Nicole Hall, Cosima Rheinwald und Susanne Seiz.

\section{$5 \quad$ Literatur}

[1] D. Kampa, A. Weiß, R.H. Schmucker: „Material Problems in Jet Vane Thrust Vector Control Systems“, AGARD Conference Proceedings No. 259 Solid Rocket Motor Technology, 1979.

[2] M. Frieß, C. Zuber, B. Heidenreich, H. Ciezki, A. Feinauer, J. van Kampen: „Keramische Verbundwerkstoffe für Flugkörper“ In: DGLR-Jahrestagung 2005, Deutsche Gesellschaft für Luft- und Raumfahrt, DGLR-Jahrestagung 2005, Friedrichshafen, 2005-09-26 - 29

[3] W. Krenkel: "Cost Effective Processing of CMC Composites by Melt Infiltration (LSI-Process)", Ceramic Engineering and Science Proceedings, Volume 22, Issue 3, p. 443-454, 2001

[4] M. Frieß, R. Renz, W. Krenkel: "Graded Ceramic Matrix Composites by LSI-Processing", Proceedings of the $10^{\text {th }}$ International Congress and $3^{\text {rd }}$ Forum on New Materials, CIMTEC, Florence, Italy, July 14-18, 2002

[5] W. Krenkel: „Entwicklung eines kostengünstigen Verfahrens zur Herstellung von Bauteilen aus keramischen Verbundwerkstoffen“, Dissertation Universität Stuttgart 2000.

[6] R. Brandt, M. Frieß, G. Neuer: "Thermal conductivity, specific heat capacity, and emissivity of ceramic matrix composites at high temperatures", 2003, High Temp. High Press. 35, 169177

[7] W. Krenkel, J. Hausherr, M. Frieß, T. Reimer: "Design, Manufacture and Quality Assurance of C/C-SiC Composites for Aeronautics and Space Transportation Systems", 28 th International Cocoa Beach Conference and Exposition on Advanced Ceramics \& Composites, Cocoa Beach, Florida, USA, 25-30.01.2004, The American Ceramic Society, (2004)

[8] M. Frieß, W. Krenkel, R. Kochendörfer, R. Brand, G. Neuer, H.P. Maier: „Ceramic matrix composites - the key materials for re-entry from space to earth", in "Basic research and technologies for two-stage-to-orbit vehicles", Ed.: D. Jacob, G. Sachs, S. Wagner, Wiley$\mathrm{VCH} /$ Weinheim, 2005, ISBN 3-527-27735-8 